\title{
Towards the operation of Cs-K-Sb photocathodes in superconducting rf photoinjectors
}

\author{
Martin A. H. Schmeißer, Sonal Mistry, Hans Kirschner, Susanne Schubert, Andreas Jankowiak, \\ Thorsten Kamps, and Julius Kühn \\ Helmholtz-Zentrum Berlin, Albert-Einstein Strasse 15, Berlin 12489, Germany
}

(Received 12 July 2018; published 19 November 2018)

\begin{abstract}
High quantum efficiency photocathodes are mandatory for the operation of photoinjector driven electron accelerators with high average current and high brightness beams. Photocathodes based on bi-alkali antimonides, e.g., $\mathrm{CsK}_{2} \mathrm{Sb}$, exhibit high quantum efficiencies for visible light and can be operated close to the photoemission threshold, thus they are suitable candidates to provide high current and low emittance electron beams. In this paper, a codeposition procedure of $\mathrm{K}$ and $\mathrm{Cs}$ on $\mathrm{Sb}$ resulting in high quantum efficiency photocathodes is presented and compared to the sequential growth procedure that was established for photomultiplier and accelerator applications. In-situ x-ray photoelectron spectroscopy is applied to gain insights into the reaction pathway of antimony with alkali metals, and to optimize the growth process of $\mathrm{CsK}_{2} \mathrm{Sb}$ on Mo. It has been found that the average stoichiometry of the samples is similar after both procedures. The study also presents the behavior of the photocurrent at cryogenic temperatures, the influence of cooling and warmup cycles on the photocathode lifetime and our experience with storage and transport. This work demonstrates that our codeposition growth procedure reproducibly delivers high quantum efficiency photocathodes, and that their quantum efficiency, when excited with green photons, is not influenced by cryogenic temperatures.
\end{abstract}

DOI: 10.1103/PhysRevAccelBeams.21.113401

\section{INTRODUCTION}

Photocathodes need to be developed as electron sources in photoinjectors for high current and continuous wave (CW) operation of energy recovery linac (ERL) and free electron laser (FEL) light sources [1]. Photocathodes for these applications require low intrinsic emittance to achieve the necessary beam brightness. The demonstration of a $100 \mathrm{~mA}$ beam with an emittance of $\varepsilon<1.0 \mathrm{~mm}$ mrad in an ERL is a main goal of the bERLinPro project [2]. One of the key challenges is to setup the prototype of the superconducting radio frequency (SRF) photoinjector, which defines the achievable brightness of the electron beam of the ERL. Firsthand experience was gained at HZB in the development of a SRF photoinjector with a lead photocathode [3]. However, for the generation of a high current and high brightness electron beam, Cs-K-Sb photocathodes are employed. Thus far Cs-K-Sb photocathodes have been used in DC photoinjectors [4-7]. Recently their performance has also been tested in an SRF photoinjector [8].

*julius.kuehn@helmholtz-berlin.de

Published by the American Physical Society under the terms of the Creative Commons Attribution 4.0 International license. Further distribution of this work must maintain attribution to the author(s) and the published article's title, journal citation, and DOI.
As new applications for photoinjectors call for an increase in brightness and lower emittance, one approach is to work at very high launch fields which allow smaller emitting spot sizes. At higher gradients, however, the surface roughness of the cathode contributes significantly to the emittance due to transverse field components.

Models suggest that at the envisaged gradients for SRF photoinjectors (15-20 MV/m) the surface roughness of the photocathode must be reduced to values of $R_{q} \leq 10 \mathrm{~nm}$ to maintain a roughness induced emittance contribution that is below the intrinsic emittance $[9,10]$. From recent works of Schubert et al. and Ding et al. it is apparent that the final roughness of alkali antimonide photocathodes depends strongly on the growth procedure and the crystallization processes involved $[11,12]$. It has been shown that the sequential growth process delivers very rough photocathodes due to the multiple phase transitions, while smoother photocathodes can be grown using new growth procedures such as triple evaporation of $\mathrm{Cs}-\mathrm{K}-\mathrm{Sb}$ using pure alkali metal sources and magnetron sputtering [13-16]. In this work an adaptation of a codeposition procedure has been developed, taking into consideration the geometrical condition of our preparation system.

Details of the two preparation procedures are presented in Secs. II and III A. Based on in-situ X-ray photoelectron spectroscopy (XPS) measurements, an investigation comparing sequentially grown $\mathrm{Cs}-\mathrm{K}-\mathrm{Sb}$ photocathodes with 
photocathodes grown by alkali metal codeposition on the antimony layer was carried out and is discussed in Sec. III B. Furthermore, the performance of the photocathodes under cryogenic temperatures was evaluated (see Sec. III C) as well as the influence of cooling on the QE lifetime (see Sec. III D). This is a critical area of research as there continues to be lively discussion within the accelerator physics photocathode community $[17,18]$ on the topic of operation under cryogenic temperatures. Experiences related to the storage and transfer of these extremely sensitive photocathodes are also presented in Secs. III D and III E.

\section{EXPERIMENTAL DETAILS}

At HZB, dedicated UHV-systems were built to produce and analyze Cs-K-Sb photocathodes for the bERLinPro project. The geometry of the sample and deposition sources is shown in Fig. 1; for details of the preparation system please refer to [19]. Recently, the system was appended by a load-lock to exchange the alkali dispensers and substrates as well as a transfer system to move samples into a vacuum suitcase for the photoinjector as detailed in [20]. The transfer chamber, substrate load-lock and vacuum suitcase are housed under a clean-room cell. The base pressures achieved are $3 \times 10^{-10}$ mbar in the preparation chamber, $7 \times 10^{-11}$ mbar in the analysis chamber and $3 \times 10^{-10} \mathrm{mbar}$ in the transfer systems. The vacuum in the suitcase for storage and transport is $5 \times 10^{-11}$ mbar.

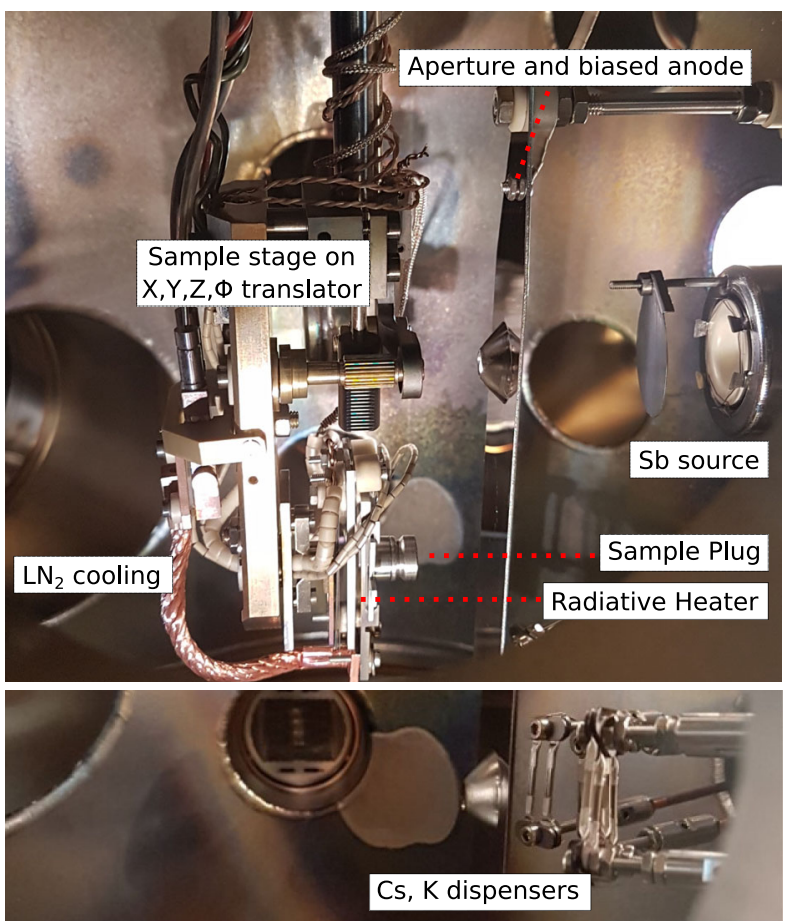

FIG. 1. Details of the geometry used for the preparation of the bi-alkali antimonide photocathodes.

\section{A. Substrate preparation}

All samples were grown on polished Mo substrates, as this is the intended plug material for photoinjector operation. Mo has also been studied in terms of unwanted field emission [21]. Mo sheets of size $10 \times 10 \mathrm{~mm}^{2}$ have been used for the sample P006 and so-called Mo plugs, with a diameter of $10 \mathrm{~mm}$ in the final photoinjector design, have been used for the other samples. In either case, the surface was polished using zirconia polishing paste to a surface roughness of approximately $\mathrm{R}_{q}=10 \mathrm{~nm}$, determined by AFM. The substrates were dry ice blown, rinsed in acetone, cleaned in an ultrasonic bath of isopropanol and transferred into UHV. In order to remove native surface oxides and residues from the polishing process, heat treatment to $450{ }^{\circ} \mathrm{C}$ and $\mathrm{Ar}^{+}$sputter cleaning at $2 \mathrm{keV}$ (performed in the preparation chamber) was found to be effective. The roughness increase due to the sputter process is less than $5 \mathrm{~nm}$ and was determined from a witness sample. The cleanliness and purity of the substrates, as well as every deposition step, could be verified by XPS in the adjacent analysis chamber.

\section{B. Preparation chamber}

$\mathrm{Sb}$ is evaporated with an effusion cell loaded with Sb-beads obtained from Alfa Aesar (99.999\% purity). The Sb-deposition rate is controlled via a quartz crystal microbalance (QMB). Cs and $\mathrm{K}$ dispenser sources from SAES are mounted separately on current feedthroughs, with an angle of $25^{\circ}$ with respect to the sample normal and a distance of $7 \mathrm{~cm}$. In this configuration $\mathrm{K}$ and $\mathrm{Cs}$ can be evaporated separately or simultaneously. The first option results in a three step procedure in which an Sb layer is formed on the substrate, then allowed to react with potassium, and finally the K-Sb interlayer is allowed to react with cesium. This procedure is termed the "traditional, sequential growth" procedure and was employed for the samples P006 and P007. The latter option is similar to begin with; an initial $\mathrm{Sb}$ layer is deposited and then allowed to react with both alkali metals, $\mathrm{K}$ and $\mathrm{Cs}$, at the same time. This procedure is therefore a two step process and termed the "alkali metal coevaporation" technique, and was used for the remaining samples P009, P013, P014, P015, P016 and G002. All other parameters are kept equal, to the extent possible, if not noted otherwise. In particular the substrate temperature during deposition, deposition rates and partial pressures of residual gas species are constant. The initial Sb film thickness is varied between 10 and $30 \mathrm{~nm}$ as discussed below. For both deposition techniques the sample is formed by diffusion of alkalis into the Sb film and the subsequent recrystallization into an alkali antimonide structure, generally of $\mathrm{M}_{3} \mathrm{Sb}$ stoichiometry where $\mathrm{M}$ is an alkali metal ion. The process is dominated by the diffusion of alkalis into the material while sufficient alkali metal vapor 
pressure must be supplied on the surface. Thus the reading of, e.g., a QMB cannot give reliable information on the total film thickness, rather relative information on the rate of metal supplied. Typically the final film thickness is about 5 times the initial Sb film thickness [22].

Before preparation all sources are carefully degassed and deposition rates are determined. Based on our own experience and measurements presented in [23], it is necessary to reach a total residual gas pressure below $5 \times 10^{-9}$ mbar and a water partial pressure below $5 \times 10^{-10}$ mbar during operation of all sources, because too high partial pressures of reactive species $\left(\mathrm{H}_{2} \mathrm{O}, \mathrm{CO}_{2}\right.$, and $\left.\mathrm{O}_{2}\right)$ will poison the sample, prevent crystallization and reduce the performance of the photocathode.

For the deposition of $\mathrm{K}$ and $\mathrm{Cs}$, a heating current of $6 \mathrm{~A}$ was applied to each dispenser. During the deposition the partial pressures are monitored with a quadrupole mass spectrometer (Pfeiffer QMA200M). For the sample preparation in both processes discussed below, the total pressure was dominated by the Hydrogen partial pressure contribution; the water vapor partial pressure never exceeded $5 \times 10^{-10} \mathrm{mbar}$, and all other species were lower still.

The growth process is followed by measuring the photocurrent extracted from the sample. For this purpose, a bias voltage of $300 \mathrm{~V}$ is applied between the pick-up anode and the sample, which is constantly illuminated with green light at $2.33 \mathrm{eV}(532 \mathrm{~nm}$; frequency doubled $\mathrm{Nd}$ : YAG laser) or $2.4 \mathrm{eV}$ (515 nm; monochromatic white light source). Note that $515 \mathrm{~nm}$ will be the available wavelength from the bERLinPro photocathode drive laser, which is the second harmonic of the original NIR radiation of a Yb:YAG laser. From the measured photocurrent the dark current is subtracted to calculate the quantum efficiency (QE), with respect to the spectral flux of the light source.

Attached to the preparation chamber is a spectral response setup, mentioned as the monochromatic white light source above [24]. The setup is used to illuminate the sample during the alkali metal evaporation to monitor the photocurrent and to measure the quantum efficiency as a function of the photon energy, ranging from $1.77 \mathrm{eV}$ $(700 \mathrm{~nm})$ to $3.35 \mathrm{eV}(370 \mathrm{~nm})$. Spectral response measurements have been used to study photocathodes under cryogenic conditions ( $\mathrm{P} 013$ and $\mathrm{P} 016$ ) and also for the following lifetime study of P016. In the case of the combined cryogenic and lifetime study, the photoemission threshold, $\phi$, has been extracted from this data according to Kirschner [25].

\section{Analysis chamber}

After each preparation step it is possible to move the sample into the analysis chamber for investigation by $\mathrm{x}$-ray photoelectron spectroscopy (XPS). The analysis chamber is directly attached to the preparation chamber and equipped with an electron analyzer (Specs Phoibos 100 2D-CCD) and an X-ray source (Specs XR 50). The spectra were acquired with medium magnification lens mode. Survey spectra were acquired with a constant pass energy of $20 \mathrm{eV}$ and detailed region spectra with $10 \mathrm{eV}$. The angle of emission between the sample surface normal and the analyzer axis was kept constant at $0^{\circ}$. XPS data were analyzed using CasaXPS and a custom PYTHON code; the quantitative analysis was performed from survey spectra $(\mathrm{Sb}$ $3 \mathrm{~d}$, Cs $3 \mathrm{~d}$, and $\mathrm{K} 2 \mathrm{p}$ regions) after Shirley background subtraction using the transmission function of the analyzer, inelastic mean free paths and Scofield cross sections [26,27].

It is important to note that alkali antimonide samples are highly reactive and oxidize within seconds when exposed to air. In order to preserve the photoemissive surface, samples must be stored and handled in UHV of few $10^{-10}$ mbar. Our vacuum suitcase is suitable to transport photocathodes to the SRF photoinjector but not to other analytical tools. Consequently, we must refer to other studies where roughness and crystallization have been studied using AFM and XRD either in-situ or after an UHV transfer. We consider the data complementary to the results presented here.

\section{RESULTS AND DISCUSSION}

Results from two samples prepared by sequential deposition (P006 and P007) as well as the results from samples grown by coevaporation of the alkali metals (P009, P013, P014, P015) are evaluated in this section. Additionally a comparison of the cool down experiments for photocathodes P013 and P016 is presented, followed by findings from a lifetime study of P016. Finally, an overview of our experiences in preparing the first photocathode for photoinjector studies is presented, focusing on growth, transport, and storage.

\section{A. Photocathode preparation}

\section{Sequential growth (3 steps)}

Good performance of sequentially deposited cathodes could be demonstrated for the following growth parameters [28,29]: deposition of a $10 \mathrm{~nm}$ thick Sb film at $0.2 \AA / \mathrm{s}$ and $100{ }^{\circ} \mathrm{C}$ substrate temperature, $\mathrm{K}$ deposition at $0.01 \AA / \mathrm{s}$ and $100^{\circ} \mathrm{C}$ until the photocurrent plateaus, Cs deposition at $0.03 \AA / \mathrm{s}$ and $90^{\circ} \mathrm{C}$ until the photocurrent plateaus. Note that the alkali rates are the maximum rates at the specified operation current. Usually each alkali deposition takes about $2 \mathrm{~h}$. The purity of the substrate and every deposition step was checked by XPS, and the $\mathrm{H}_{2} \mathrm{O}$ partial pressure never exceeded $5 \times 10^{-10}$ mbar. After preparation, sample P006 had a quantum efficiency of $4.8 \%$ at $2.33 \mathrm{eV}$, while P007 had a QE of $1.6 \%$ at $2.33 \mathrm{eV}$.

\section{Alkali metal codeposition growth (2 steps)}

The codeposition of the photocathode samples was performed in a similar manner. The Sb film was deposited 

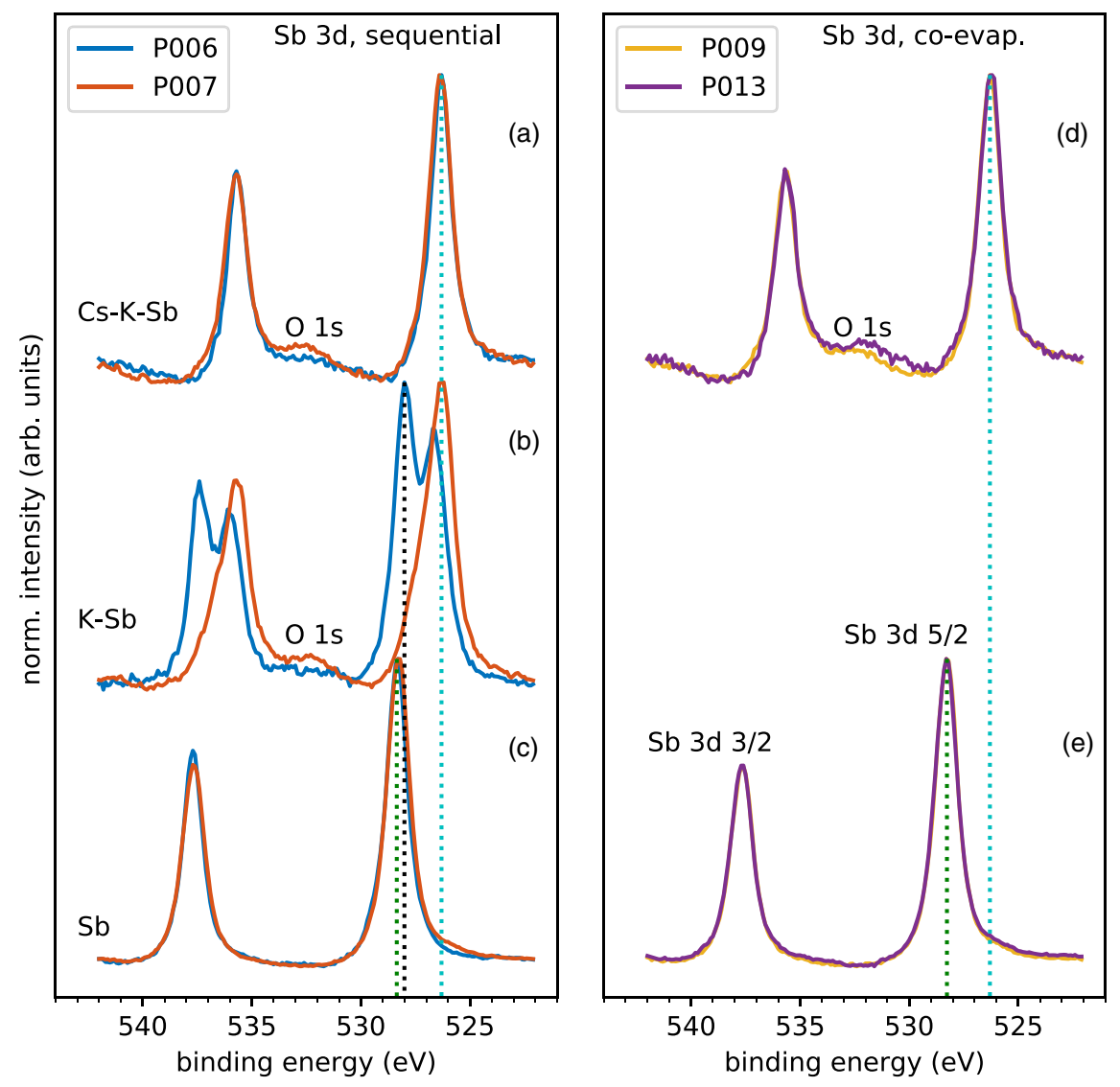

FIG. 2. Comparison of the Sb 3d spectra of four samples, two grown sequentially (left) and two by alkali metal coevaporation (right). In both processes the alkali metals were evaporated on a pure Sb layer (c, e). The Sb 3d spectrum of the K-Sb layer of P006 shows two peaks, indicating that the Sb has not fully reacted with $\mathrm{K}$ (b, blue line). The spectra of the Cs-K-Sb layers are identical for all samples (a and d).

first, using the same parameters as in the sequential procedure. Subsequently, the alkali metals were codeposited simultaneously using the operation currents determined using a QMB prior to the deposition. During this procedure the $\mathrm{H}_{2} \mathrm{O}$ partial pressure never exceeded $5 \times 10^{-10}$ mbar. The alkali metal codeposition was terminated when a plateau in the photocurrent was observed. After preparation the photocathodes P009 and P013 had a $\mathrm{QE}$ of $2.6 \%$ and $5.6 \%$ at $2.33 \mathrm{eV}$.

\section{B. Comparison of growth procedures by XPS}

In this section the chemical analysis by XPS of the two different growth procedures is presented: $\mathrm{P} 006$ and P007 for the sequential growth and P009 and P013 for the alkali metal codeposition on $\mathrm{Sb}$.

Figure 2 shows the $\mathrm{Sb} 3 \mathrm{~d}$ region spectra for each preparation step. For all samples a pure $\mathrm{Sb}$ layer was grown on polished, pure and clean Mo substrates. No signal from the Mo substrate was discernible after any deposition step.

Figure 2(c) and (e) show that after the first step, the $\mathrm{Sb}$ $3 \mathrm{~d}_{5 / 2}$ peak was found at $528.3 \mathrm{eV}$ and the $\mathrm{Sb} 3 \mathrm{~d}_{3 / 2}$ at
$536.7 \mathrm{eV}$, corresponding to $\mathrm{Sb}(0)$. The next step for P006 and P007 was K-deposition on the Sb-layer as shown in Fig. 2(b); for P006 this resulted in a mixed K-Sb phase. The dominant $\mathrm{Sb} 3 \mathrm{~d}_{5 / 2}$ peak at $528.0 \mathrm{eV}$ is related to $\mathrm{K}$ deposited on $\mathrm{Sb}$, while the second peak with lower intensity at $526.6 \mathrm{eV}$ is indicative of the $\mathrm{Sb}(3-)$ state of $\mathrm{K}_{3} \mathrm{Sb}$.

Finally for P006 and P007, Cs was deposited on the $\mathrm{K}-\mathrm{Sb}$ film reacting to a Cs-K-Sb photocathode [Fig. 2(a)]. For P006, the $\mathrm{Sb}(3-) 3 \mathrm{~d}_{5 / 2}$ peak shifted to $526.3 \mathrm{eV}$, while the $\operatorname{Sb}(0) 3 \mathrm{~d}_{5 / 2}$ disappeared.

The composition of both the intermediate K-Sb layers of P006 and P007 as determined by XPS show a K deficiency with respect to $\mathrm{K}_{3} \mathrm{Sb}$ (see Table I). Nevertheless, the almost rigid shift of the $\mathrm{Sb} 3 \mathrm{~d}$ peak of $\mathrm{P} 007$ indicates that most antimony in the probe depth has reacted (peaks at $526.3 \mathrm{eV}$ and $535.6 \mathrm{eV}$, red line). A shoulder is discernible in the $\mathrm{Sb} 3 \mathrm{~d} 5 / 2$ peak for $\mathrm{P} 007$ which hints at traces of nonreacted $\mathrm{Sb}$. In contrast, the double structure in the $\mathrm{Sb} 3 \mathrm{~d}$ peak of P006 indicates the presence of nonreacted $\mathrm{Sb}$ (peaks at $528.3 \mathrm{eV}$ and $536.7 \mathrm{eV}$ ) as well as reacted $\mathrm{Sb}$ in the $\mathrm{Sb}(3-)$ state. The effect of different levels of reaction will be discussed below. The final Cs-K-Sb photocathode layers of P006, P007, P009, and P0013 have almost identical Sb 3d 
TABLE I. Chemical composition, thickness of the initial Sb layer, and final QE of the sequentially and codeposited samples. The QE is measured at $2.33 \mathrm{eV}$, as prepared.

\begin{tabular}{lcccccc}
\hline \hline Sample & & Sb layer (nm) & Sb & K & Cs & QE (\%) \\
\hline P006 & K-Sb & 10 & 1 & 2.3 & & \\
& Cs-K-Sb & & 1 & 1.8 & 1.4 & 4.8 \\
P007 & K-Sb & 10 & 1 & 2.7 & & \\
& Cs-K-Sb & & 1 & 2.4 & 0.8 & 1.6 \\
P009 & Cs-K-Sb & 10 & 1 & 1.9 & 1.4 & 2.6 \\
P013 & Cs-K-Sb & 30 & 1 & 1.5 & 2.3 & 5.6 \\
P014 & Cs-K-Sb & 30 & 1 & 0.5 & 1.8 & 7.7 \\
P015 & Cs-K-Sb & 30 & 1 & 1.0 & 2.3 & 7.2 \\
\hline \hline
\end{tabular}

peak shapes which are fully converted to the $\mathrm{Sb}(3-)$ state [Fig. 2(a) and 2(d)].

Figure 3 presents the $K 2 p$ and $C s 3 d$ spectra of the respective samples. Figure 3(c) shows the $\mathrm{K} 2 \mathrm{p}$ spectrum of P006 and P007 after the second step (K-Sb); for P006 the $\mathrm{K} 2 \mathrm{p}_{3 / 2}$ peak is at a binding energy of $293.7 \mathrm{eV}$ and the $\mathrm{K} 2 \mathrm{p}_{1 / 2}$ is at $296.5 \mathrm{eV}$. The $\mathrm{K} 2 \mathrm{p}$ peaks are shifted by approximately $0.4 \mathrm{eV}$ to lower binding energies after the Cs deposition [Fig. 3(b)]; this is now the same position compared to the K $2 \mathrm{p}$ spectra of the other samples. The Cs $3 \mathrm{~d}$ spectra in Fig. 3 are almost identical for all samples. The Cs $3 \mathrm{~d}_{5 / 2}$ in Cs-K-Sb is at a binding energy of $725.5 \mathrm{eV}$ and the $\mathrm{Cs} 3 \mathrm{~d}_{3 / 2}$ at $739.2 \mathrm{eV}$.

The measured spectra-Cs 3d, K 2p, and Sb 3d-have equal binding energies and peak shapes for the final $\mathrm{Cs}-\mathrm{K}-\mathrm{Sb}$ films of all samples regardless of the slight differences in the stoichiometry. In Fig. 2(a) and (d), the $\mathrm{Sb} 3 \mathrm{~d}$ spectrum of P007, P009, and P013, a weak O 1s peak is discernible at about $532.6 \mathrm{eV}$, which might originate from residual gases or from the $\mathrm{K}$ and Cs dispensers. It is also observed in the intermediate K-Sb layer of P007 [Fig. 3(b)], but no oxygen was observed in the pure $\mathrm{Sb}$ layers. The presence of oxygen may be an additional factor contributing to the lower QE values for these samples.

All measured binding energies are in good agreement with a previous XPS study by Schubert et al. performed in a different laboratory [29].

The present data suggests, that the full crystallization of a $\mathrm{K}_{3} \mathrm{Sb}$ layer, as evidenced in P007, leads to formation of a stable phase prior to the final preparation step. Thus recrystallization into a $\mathrm{Cs}_{2} \mathrm{KSb}$ or $\mathrm{CsK}_{2} \mathrm{Sb}$ lattice that incorporates the much larger $\mathrm{Cs}$ atoms is energetically unfavorable, leading to the low performance of a sample like P007. It is possible in the sequential technique to grow high QE samples when the intermediate K-Sb phase does not fully react and thus stays more susceptible to diffusion and recrystallization during the cesiation step, as observed with sample P006. The hypothesis is supported by XRD data presented by Schubert et al. [11,30]. The formation of a stable hexagonal $\mathrm{K}_{3} \mathrm{Sb}$ phase is witnessed as a last step during the $\mathrm{K}$ deposition after formation of the cubic phase.
The temperature dependence observed in their data shows that the recrystallization of the hexagonal phase is unfavorable compared to the cubic phase. Co-evaporation of both alkali metals avoids this problem because the formation of the hexagonal phase seems unlikely considering that it develops last in the $\mathrm{K}$ deposition of a sequential growth. Here, only a single crystallization into the cubic Cs-K-Sb lattice takes place which is also favorable in terms of surface roughness.

The chemical composition of six photocathodes in total were analyzed based on XPS data. The stoichiometry of the samples in Table I was determined from the relative peak intensity of the $\mathrm{Sb} 3 \mathrm{~d}, \mathrm{~K} \mathrm{2p}$ and $\mathrm{Cs} 3 \mathrm{~d}$ peaks taken from survey spectra. The quantification model assumes a homogeneous depth distribution of the atomic concentrations, which have been normalized to $\mathrm{Sb}$. For the sequential growth (P006, P007), the composition of the K-Sb layer is also given. The rest of the photocathodes presented in Table I have been grown in two steps. The quantitative data of P014 and P015 additionally demonstrates the reproducibility of our growth process with respect to high QE. Our data supports the claim that higher stoichiometric content of Cs, e.g., $\mathrm{Cs}_{2} \mathrm{KSb}$, is favorable for a higher QE relative to the $\mathrm{CsK}_{2} \mathrm{Sb}$ lattice. This agrees with the theoretical findings by Ettema and de Groot [31].

The thickness of the Sb layer is also presented in Table I. High QE samples have been achieved by tuning the initial thickness of the Sb-film. Furthermore a thicker film is known to be correlated to a longer QE lifetime [32].

\section{Cryogenic temperature study}

In the case of the SRF photoinjector for bERLinPro, the photocathodes will be operated at $80 \mathrm{~K}$ within the $1.8 \mathrm{~K}$ cavity back wall. Thus, the effect of temperature reduction on the spectral QE of a photocathode is a topic of great interest for SRF photoinjectors. The photocathode must be cooled to (a) reduce thermal radiation in the cavity and (b) mitigate the heat load from rf surface currents and the photocathode drive laser power. Furthermore, it is expected that the transverse energy distribution of photoelectrons becomes dominated by the lattice temperature when the excitation energy is lowered close to the photoemission threshold and, thus, ultracold electron beams can only be emitted from cryocooled cathodes [33]. However, a reduction of QE or an increase in work function at cryogenic temperatures have been reported and are currently subject to much discussion within the community. This would lead to a trade-off situation where low emittances due to cooling can only be obtained at the price of reduced QE.

Cooling tests with two photocathodes, P013 and P016, have been performed in the preparation chamber in order to observe the photocathode performance in terms of $\mathrm{QE}$. Both photocathodes were cooled with liquid nitrogen using the manipulator in the preparation chamber, which is also 

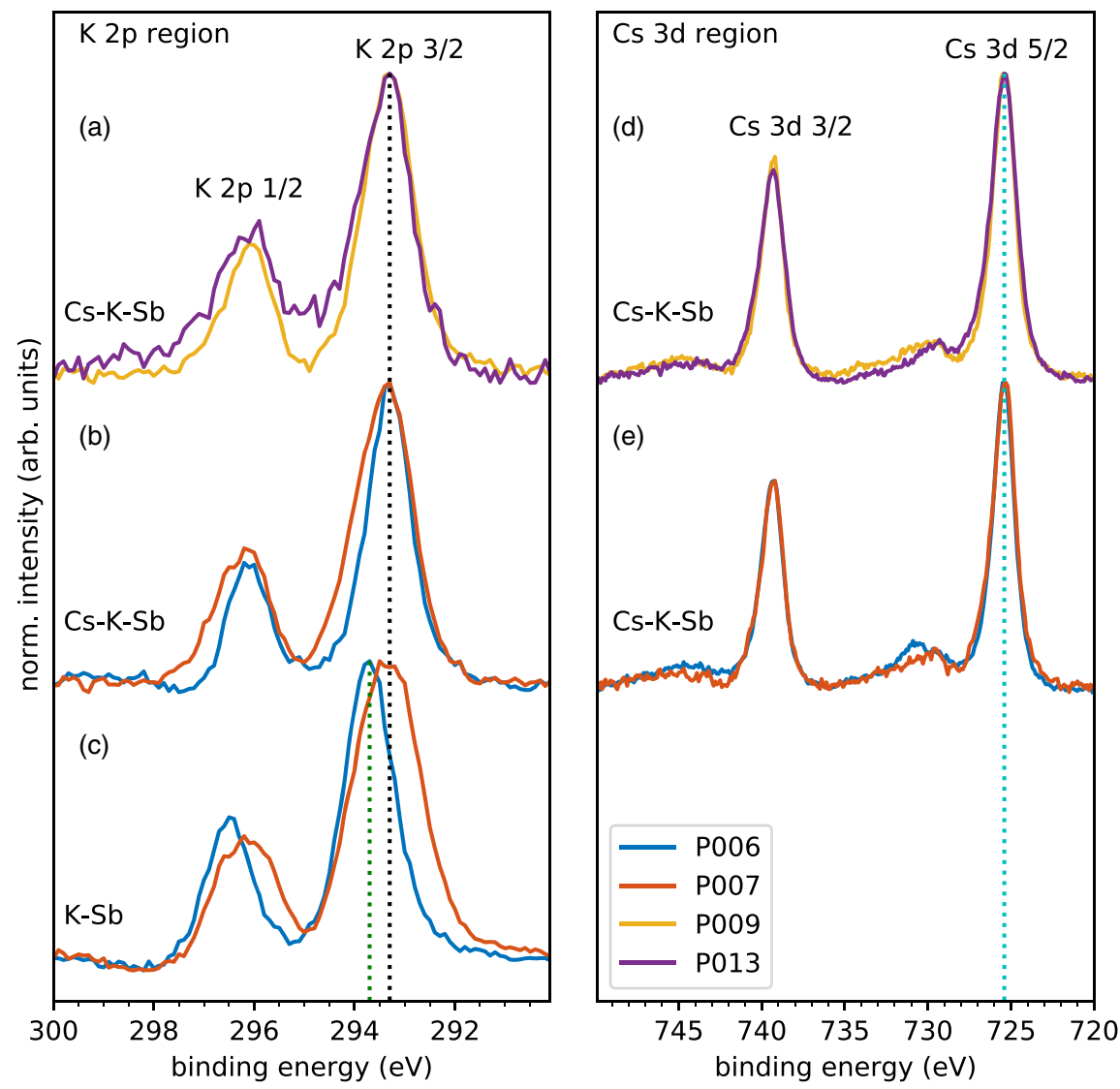

FIG. 3. Comparison of the K 2p (left) and Cs 3d (right) spectra of each sample. The color code holds for both panels. Note the shift in the P006 K 2p spectra between the K-Sb and Cs-K-Sb layer (b and c, blue lines). The K 2p and Cs 3d spectra of the Cs-K-Sb layer of the other samples show no difference in terms of binding energy and peak shapes (a and $\mathrm{d}$ ).

used for the sample preparation. The effective cooling for each photocathode varied slightly due to minor difference in the nitrogen gas flow and also in the thermal contact of the copper cooling wire to the sample stage.

The characteristic spectral response plots are shown in Fig. 4. No strong reduction of the QE at $2.4 \mathrm{eV}$ was observed when P013 was cooled from room temperature to $150 \mathrm{~K}$. The measured photocurrent remained nearly constant and the maximum vacuum pressure observed was $2 \times 10^{-10}$ mbar. A significant decrease in QE was observed when the cold sample was moved into the analysis chamber (in vacuum); this resulted in an increase in pressure above $1 \times 10^{-9}$ mbar. The warm up of P013 back to RT did not show either a decrease or recovery of the QE. To reduce the risk of increasing the vacuum pressure, movement within the system was avoided in the case of P016. Consequently the pressure stayed constantly below $6 \times 10^{-10}$ mbar. There is very little discernible difference in the spectral response between the warm and cold sample (Fig. 4, P016, A, and B). The decrease of the $\mathrm{QE}$ observed in the following measurements $(\mathrm{C}, \mathrm{D}, \mathrm{E})$ is related to fatigue of the sample, which is presented and discussed in Sec. III D.
For the purpose of this discussion, the relative sensitivity, $S_{\text {rel }}(\lambda)$, is defined as the fraction of the spectral response at room temperature and the cryocooled sample:

$$
S_{\text {rel }}(\lambda)=\frac{Q E_{\text {cryo }}(\lambda)}{Q E_{\mathrm{RT}}(\lambda)}
$$

The corresponding plots shown in Fig. 5 are deduced from the spectral response measurements during the thermal cycles displayed in Fig. 4. For P013, two relative sensitivities are deduced from one cycle as a ratio of the spectral response measurements before and after the cooldown as well as the ratio before and after letting the sample warm up. The two ratios shown for P016 are obtained from the cooldown of two consecutive cycles. The format of Fig. 5 has been chosen for ease of comparison to the values reported in the literature, e.g., [34]. Notably, for both samples the relative sensitivity differs strongly between the first thermal cycle (blue lines) and the second thermal cycle (red lines). The spectral sensitivity during the first cycle shows values greater than unity, and yet there is no clear trend nor similar behavior between the two samples. The difference in behavior can perhaps be attributed to the 


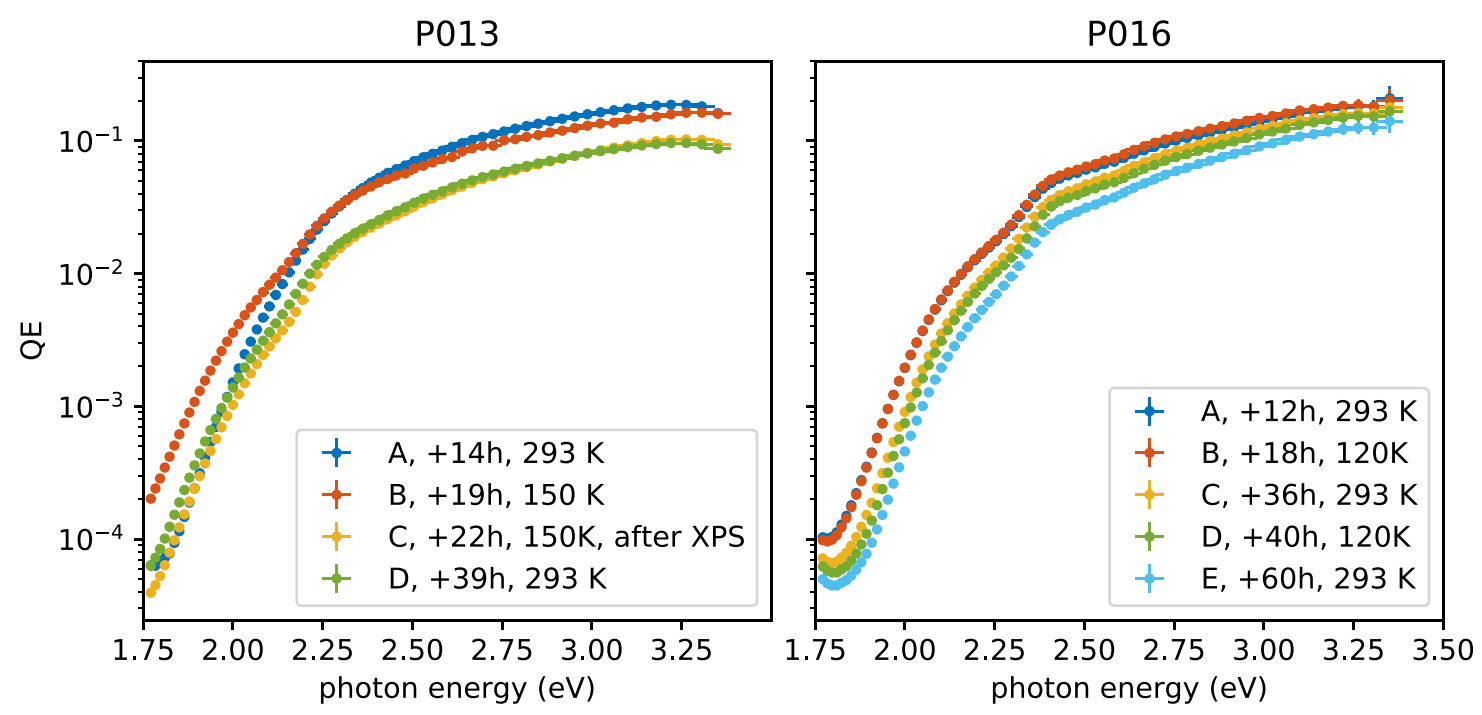

FIG. 4. Spectral response as obtained during the cryogenic cooling experiments with P013 and P016 with a vacuum pressure in the low $10^{-10}$ mbar regime. P013 was cooled down to $150 \mathrm{~K}$ while P016 was cooled repeatedly to $120 \mathrm{~K}$. Both experiments were performed in the photocathode preparation chamber and cooling of the sample stage with liquid nitrogen. The age of each sample is indicated relative to the QE measurements presented in Table I.
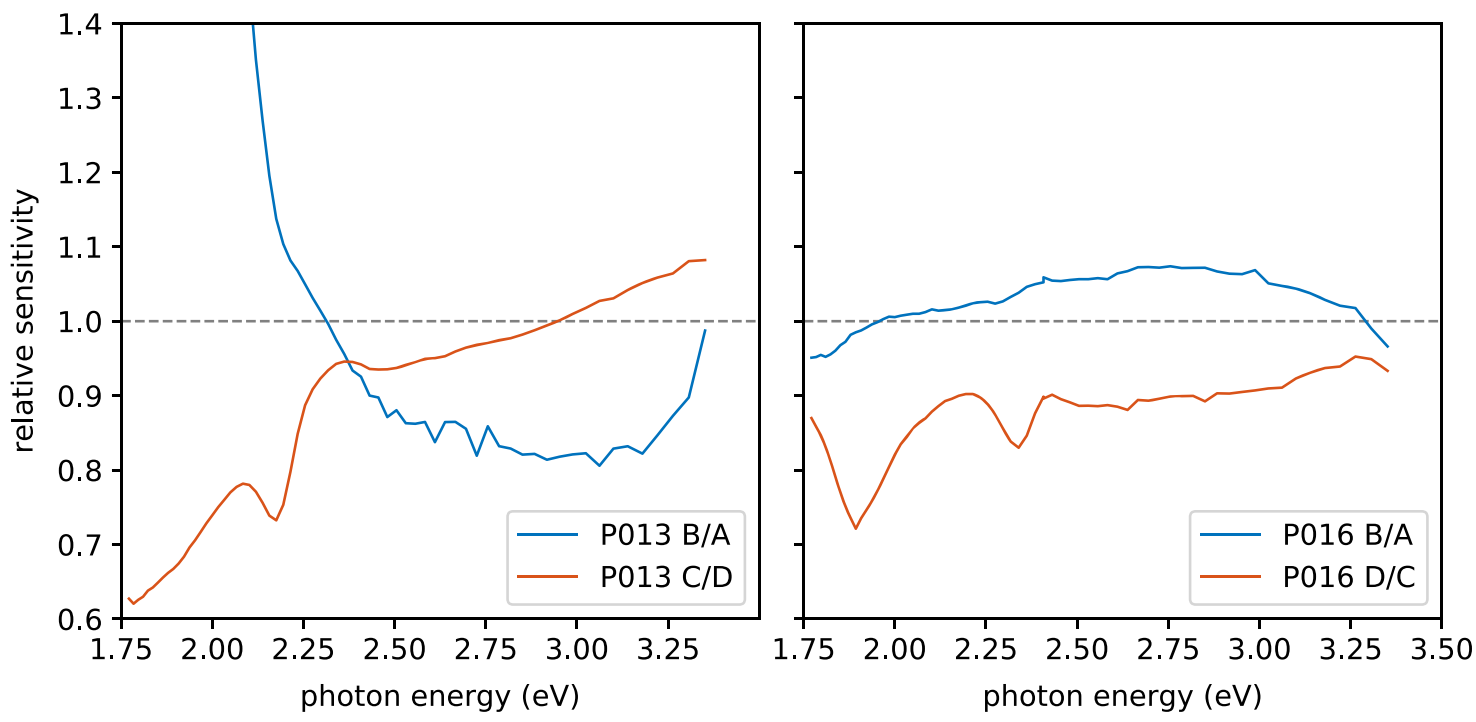

FIG. 5. Relative sensitivity of the samples P013 and P016 during the various cycles. The horizontal line at unity is to guide the eye.

fact that during the first thermal cycle, the photocathode is still active - the alkali metals would still be diffusing into the $\mathrm{Sb}$ layer, and residual gas molecules would be adsorbing on the pristine surface. This dynamic environment contrasts with the relatively saturated surface found during the second cycle. The pronounced increase in the lowenergy response shown for P013 B/A has also been reported in [23] after a slight exposure $(<2 \mathrm{~L})$ to Oxygen, and is also found in Fig. 7. We therefore attribute this effect to the adsorption of oxygen on the surface and formation of a surface dipole, in this case intensified by the larger sticking coefficient of the cold surface.
Considering the relative sensitivity measurements from the second cycle, it is evident that the results are in agreement; they indicate that the spectral sensitivity reduces with lower photon energy. One may assume that the reduction in $\mathrm{QE}$ for low photon energies can be explained by a reduced number of states populated by the tail of the Fermi-Dirac distribution which contribute to photoemission at room temperature but are less populated at cryogenic temperatures. At the same time the number of scattering partners for the electrons excited by higher photon energies is reduced, thus the mean free path and $\mathrm{QE}$ are increased; this can explain values greater than unity 
or a less pronounced loss of $\mathrm{QE}$ for excitation energies greater than $2.5 \mathrm{eV}$. Yet, such general consideration cannot explain the oscillatory behavior observed in the range of $2.0 \mathrm{eV}$ and $2.5 \mathrm{eV}$ (red lines in Fig. 5) which necessitates a much more detailed treatment, taking into account the full band structure and scattering during the emission process; such models are currently being developed by $[35,36]$. The measurements presented by Menegolli et al. and Nathan et al. agree with our results presented here by the fact that the $\mathrm{QE}$ at approximately $2.4 \mathrm{eV}$ remains constant with temperature $[34,37]$.

Our experiments indicate that the operation of a Cs-K-Sb photocathode at $80 \mathrm{~K}$ is possible without compromising the QE. Though the vacuum conditions in the SRF photoinjector are still unknown, they are expected to be even better than in the preparation chamber, because of the enhanced cryogenic pumping performance of the $\mathrm{Nb}$-cavity at $1.8 \mathrm{~K}$.

\section{Lifetime study}

In addition to the cooling cycles, the lifetime of the photocathode P016 was monitored. After preparation, P016 was not moved within the preparation and analysis system to avoid degradation, hence no XPS data is available for this sample. According to Fig. 6 the cooling and warm up cycles did not lead to significant acceleration of the degradation process, and the separate values taken from spectral response measurements line up with the exponential decay that progresses independently of the temperature.

A $1 / e$ lifetime of $50.7 \mathrm{~h}$ was determined, by illuminating the photocathode with green light $(2.4 \mathrm{eV})$ over more than $120 \mathrm{~h}$. The constant illumination was paused at several points to acquire the spectral response and the corresponding points are highlighted in Fig. 6. Along with the QE, the values for the photoemission threshold are shown.

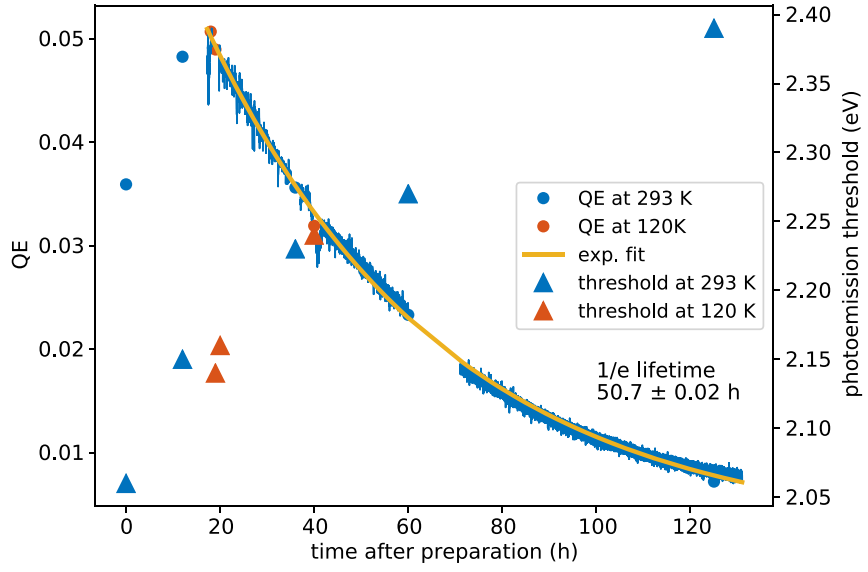

FIG. 6. Lifetime in terms of QE at $2.4 \mathrm{eV}$ of sample P016. The data points are extracted from spectral response measurements while the blue curve represents a continuous measurement.
To determine the photoemission threshold, the spectral QE shown in Fig. 4 is approximated to a linear function [25]:

$$
Q E(\nu)=A(h \nu-\phi)
$$

where $\mathrm{A}$ is a constant equivalent to the gradient, and $\phi$ is defined as the photoemission threshold and is determined from the $\mathrm{x}$-intercept.

In the first $19 \mathrm{~h}$ after preparation, the QE continues to increase due to the ongoing reactions in the photocathode layer. After this period, the QE decreases, and an increase of the photoemission threshold is observed; this is likely related to the adsorption of residual gas molecules and their reaction with the highly reactive photocathode layer [38]. By the end of the measurements $(125 \mathrm{~h})$ the $\mathrm{QE}$ reached $0.7 \%$ and the threshold calculated was found to be $2.39 \mathrm{eV}$. It is interesting to note that this value is comparable with the energy of the drive laser for bERLinPro $(2.4 \mathrm{eV} ; 515 \mathrm{~nm})$.

\section{E. Storage, transport and insertion}

Due to the finite lifetime of Cs-K-Sb photocathodes and other semiconducting candidates, an exchange mechanism for the spent photocathode must be foreseen in the injector. However, the challenges in the engineering of the exchangeable cathode interface to the SRF gun are that the normal conducting cathode cannot be in contact with the superconductor, both to avoid thermal quenching of the cavity as well as to disconnect the cathode from surface currents flowing in the cavity wall. Furthermore, particulate-free cleaning and assembly of all components must be ensured in order to mitigate particulate contamination of the cathode and cavity. At HZB a photocathode plug transfer chain has been commissioned with particulatefree vacuum conditions in the low $10^{-10}$ mbar range [20].

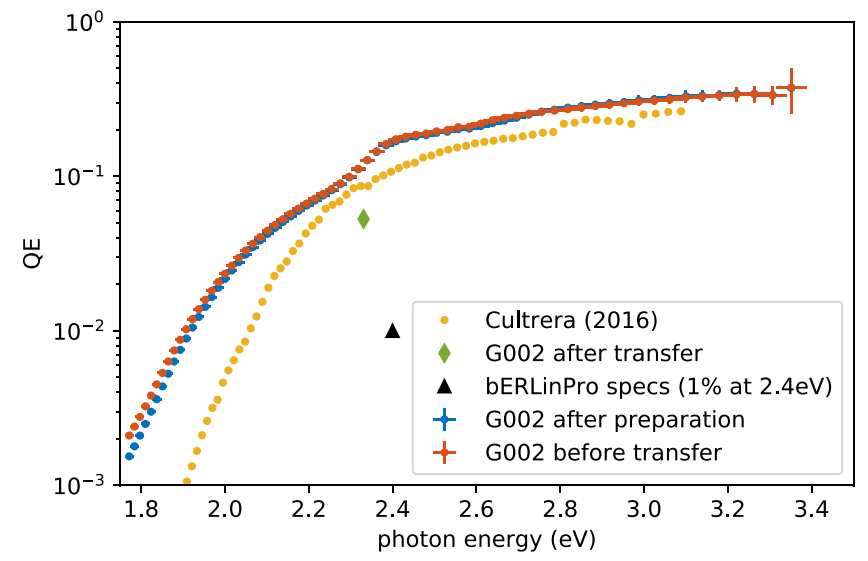

FIG. 7. Spectral response measurements of the Cs-K-Sb photocathode (G002) prepared for the SRF photoinjector operation, after preparation (blue) and before transport (red). The green diamond shows the QE after transportation and the black triangle indicates the QE specification for bERLinPro. The reference data was obtained from Cultrera et al. [39]. 

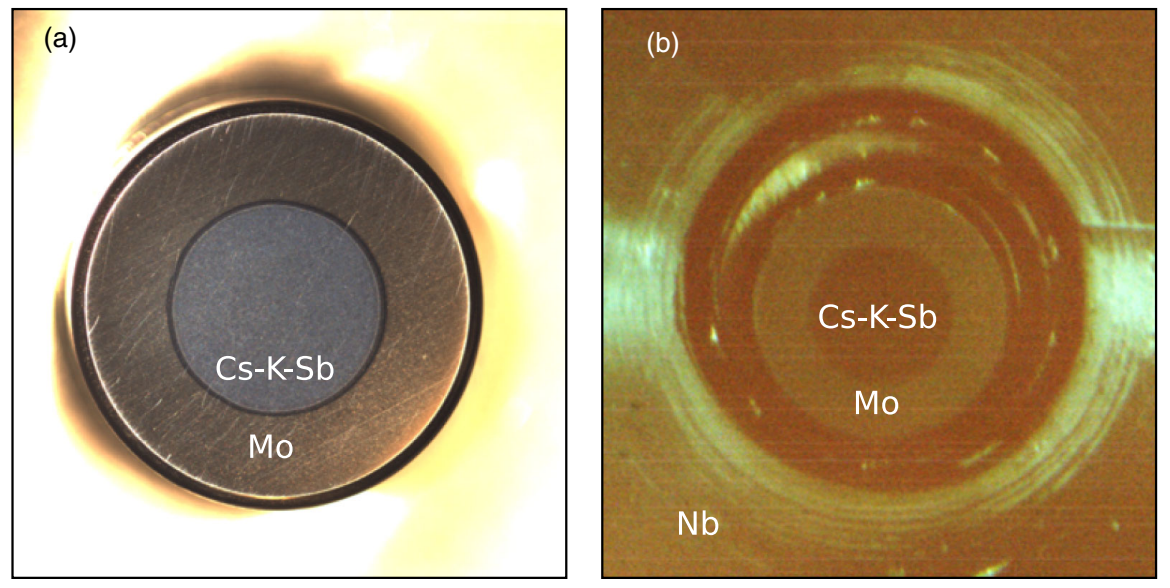

FIG. 8. Images of G002 taken (a) after growth in the preparation chamber and (b) after transfer into the SRF photoinjector.

The system allows the transport and storage of three photocathodes, plus one location for the active cathode. To demonstrate a successful transfer, a photocathode (G002) was grown in the preparation system, transferred to the vacuum suitcase (low $10^{-11} \mathrm{mbar}$ ), stored in darkness for six days and then transferred to the photoinjector transfer chamber. The QE achieved for this photocathode after preparation was $12.7 \%$ at $2.33 \mathrm{eV}$. Thus far, this is the highest QE photocathode prepared in our preparation system using the alkali metal codeposition growth recipe. Once the photocathode had been transferred into the photoinjector transfer chamber, the QE measured was $5.3 \%$. The spectral response of this sample is shown in Fig. 7 together with reference data obtained from the literature [39] and the specifications for bERLinPro.

Figure 8 shows two camera images of the photocathode (a) right after growth in the preparation chamber and (b) at its final position in the SRF-photoinjector. The image shows that the photocathode film is centered on the Mo plug and is also visible against the Nb-cavity back wall. Due to a malfunction of the cathode insert after operation in the gun with a copper photocathode, the semiconductor photocathode could not be tested [40]. Nevertheless we have demonstrated the growth of a high QE Cs-K-Sb photocathode which was stable enough to survive the transfer under vacuum and arrive at the photoinjector with a QE greater than 1\% as required for bERLinPro.

\section{CONCLUSION}

A two-step growth procedure of Cs-K-Sb photocathodes was developed with respect to our preparation system and systematically studied by XPS. The coevaporation of alkali metals on an Sb film is a reproducible method that yields high quality photocathode samples. The more recent samples were grown on a Mo plug in a geometry that can be used in the photoinjector; this is an essential step forward towards using them in the bERLinPro SRF-photoinjector. Further contributions have been made to the discussion over photocathode performance at cryogenic temperatures through our cryogenic temperature study. There was no immediate degradation in the $\mathrm{QE}$ at green, and nor did multiple cooling and warm-up cycles appear to significantly influence the lifetime of the photocathode. The growth, storage and transfer of a photocathode, with a QE above the specifications for bERLinPro, has been successfully demonstrated in this work. This is a very promising result for their ultimate use in a photoinjector. There remain additional challenges such as the cooling of the cathode insert and its mechanical stability. Therefore, further engineering and research is in progress to ensure that the cathode insert can withstand the heat load from high gradient rf fields and from the photocathode drive laser during operation.

\section{ACKNOWLEDGMENTS}

The work is partly funded by grants of the state of Berlin, the Helmholtz Association, and the European Commission (EuCARD-2 GA 312453). Furthermore we would like to acknowledge with much appreciation the crucial role of John Smedley and his group at Brookhaven National Laboratory, NY, USA, for sharing their experiences in photocathode growth.

[1] P. Musumeci, J. G. Navarro, J. Rosenzweig, L. Cultrera, I. Bazarov, J. Maxson, S. Karkare, and H. Padmore, Advances in bright electron sources, Nucl. Instrum. Methods Phys. Res., Sect. A 907, 209 (2018).

[2] M. Abo-Bakr et al., in Proceedings of IPAC 2018 (JACoW, Vancouver, BC, 2018), pp. 4127-4130.

[3] R. Barday, A. Burrill, A. Jankowiak, T. Kamps, J. Knobloch, O. Kugeler, A. Matveenko, A. Neumann, M. Schmeisser, J. Voelker, P. Kneisel, R. Nietubyc, S. Schubert, J. Smedley, J. Sekutowicz, and I. Will, Characterization of a superconducting $\mathrm{Pb}$ photocathode in a superconducting rf photoinjector cavity, Phys. Rev. Accel. Beams 16, 123402 (2013). 
[4] D. H. Dowell, K. J. Davis, K. D. Friddell, E. L. Tyson, C. A. Lancaster, L. Milliman, R. E. Rodenburg, T. Aas, M. Bemes, S. Z. Bethel, P. E. Johnson, K. Murphy, C. Whelen, G. E. Busch, and D. K. Remelius, First operation of a photocathode radio frequency gun injector at high duty factor, Appl. Phys. Lett. 63, 2035 (1993).

[5] L. Cultrera, J. Maxson, I. Bazarov, S. Belomestnykh, J. Dobbins, B. Dunham, S. Karkare, R. Kaplan, V. Kostroun, Y. Li, X. Liu, F. Löhl, K. Smolenski, Z. Zhao, D. Rice, P. Quigley, M. Tigner, V. Veshcherevich, K. Finkelstein, D. Dale et al., Photocathode behavior during high current running in the Cornell energy recovery linac photoinjector, Phys. Rev. Accel. Beams 14, 120101 (2011).

[6] B. Dunham et al., Record high-average current from a high-brightness photoinjector, Appl. Phys. Lett. 102, 034105 (2013).

[7] R. R. Mammei, R. Suleiman, J. Feingold, P. A. Adderley, J. Clark, S. Covert, J. Grames, J. Hansknecht, D. Machie, M. Poelker, T. Rao, J. Smedley, J. Walsh, J. L. McCarter, and M. Ruiz-Osés, Charge lifetime measurements at high average current using a $K_{2} \mathrm{CsSb}$ photocathode inside a dc high voltage photogun, Phys. Rev. Accel. Beams 16, 033401 (2013).

[8] I. Pinayev et al., in Proceedings of IPAC 2016 (JACoW, Busan, 2016), pp. 2085-2087.

[9] T. Vecchione, J. Feng, W. Wan, H. A. Padmore, I. Ben-Zvi, X. Liang, M. Ruiz-Osés, T. Rao, J. Smedley, and D. Dowell, in Proceedings of the 3rd International Particle Accelerator Conference, New Orleans, LA, 2012 (IEEE, Piscataway, NJ, 2012), p. 655.

[10] H. J. Qian, C. Li, Y. C. Du, L. X. Yan, J. F. Hua, W. H. Huang, and C.X. Tang, Experimental investigation of thermal emittance components of copper photocathode, Phys. Rev. Accel. Beams 15, 040102 (2012).

[11] S. Schubert, J. Wong, J. Feng, S. Karkare, H. Padmore, M. Ruiz-Osés, J. Smedley, E. Muller, Z. Ding, M. Gaowei, K. Attenkofer, X. Liang, J. Xie, and J. Kuehn, Bi-alkali antimonide photocathode growth: An X-ray diffraction study, J. Appl. Phys. 120, 035303 (2016).

[12] Z. Ding, M. Gaowei, J. Sinsheimer, J. Xie, S. Schubert, H. Padmore, E. Muller, and J. Smedley, In-situ synchrotron x-ray characterization of $K_{2} \mathrm{CsSb}$ photocathode grown by ternary co-evaporation, J. Appl. Phys. 121, 055305 (2017).

[13] M. Ruiz-Osés, S. Schubert, K. Attenkofer, I. Ben-Zvi, X. Liang, E. Muller, H. Padmore, T. Rao, T. Vecchione, J. Wong, J. Xie, and J. Smedley, Direct observation of bi-alkali antimonide photocathodes growth via in operando x-ray diffraction studies, APL Mater. 2, 121101 (2014).

[14] S. Schubert, J. Wong, J. Feng, S. Karkare, H. Padmore, M. Ruiz-Osés, J. Smedley, E. Muller, Z. Ding, M. Gaowei, K. Attenkofer, X. Liang, J. Xie, and J. Kuehn, Bi-alkali antimonide photocathode growth: An X-ray diffraction study, J. Appl. Phys. 120, 035303 (2016).

[15] J. Feng, S. Karkare, J. Nasiatka, S. Schubert, J. Smedley, and H. Padmore, Near atomically smooth alkali antimonide photocathode thin films, J. Appl. Phys. 121, 044904 (2017).

[16] M. Gaowei, Z. Ding, S. Schubert, H. B. Bhandari, J. Sinsheimer, J. Kuehn, V. V. Nagarkar, M. S. J. Marshall, J. Walsh, E. M. Muller, K. Attenkofer, H. J. Frisch, H. Padmore, and J. Smedley, Synthesis and X-ray charac- terization of sputtered bi-alkali antimonide photocathodes, APL Mater. 5, 116104 (2017).

[17] H. Xie, I. Ben-Zvi, T. Rao, T. Xin, and E. Wang, Experimental measurements and theoretical model of the cryogenic performance of bialkali photocathode and characterization with Monte Carlo simulation, Phys. Rev. Accel. Beams 19, 103401 (2016).

[18] M. Mamun, M. Hernandez-Flores, E. Morales, C. Hernandez-Garcia, and M. Poelker, Temperature dependence of alkali-antimonide photocathodes: Evaluation at cryogenic temperatures, Phys. Rev. Accel. Beams 20, 103403 (2017).

[19] M. A. H. Schmeißer, A. Jankowiak, T. Kamps, and J. Kuehn, in Proceedings of ERL 2015 (JACoW, Stony Brook, NY, 2015), pp. 97-99.

[20] J. Kuehn, J. Borninkhof, M. Bürger, A. Frahm, A. Jankowiak, T. Kamps, M. A. H. Schmeißer, M. Schuster, P. Murcek, J. Teichert, and R. Xiang, in Proceedings of IPAC 2017 (JACoW, Kopenhagen, 2017), pp. 1381-1383.

[21] S. Lagotzky, R. Barday, A. Jankowiak, T. Kamps, C. Klimm, J. Knobloch, G. Müller, B. Senkovskiy, and F. Siewert, Prevention of electron field emission from molybdenum substrates for photocathodes by the native oxide layer, Eur, Phys. J. Appl. Phys. 70, 21301 (2015).

[22] J. Xie, M. Demarteau, R. Wagner, S. Schubert, M. Gaowei, K. Attenkofer, J. Walsh, J. Smedley, J. Wong, J. Feng, H. Padmore, M. Ruiz-Osés, Z. Ding, X. Liang, E. Muller, and I. Ben-Zvi, Synchrotron X-ray study of a low roughness and high efficiency $\mathrm{K} 2 \mathrm{CsSb}$ photocathode during film growth, J. Phys. D 50, 205303 (2017).

[23] A. di Bona, F. Sabary, S. Joly, P. Michelato, D. Sertore, C. Pagani, and S. Valeri, Development, operation and analysis of bialkali antimonide photocathodes for high-brightness photo-injectors, Nucl. Instrum. Methods Phys. Res., Sect. A 385, 385 (1997).

[24] H. Kirschner, A. Jankowiak, T. Kamps, J. Kuehn, and M. A. H. Schmeißer, in Proceedings of IPAC 2017 (JACoW, Kopenhagen, 2017), pp. 1378-1380.

[25] H. Kirschner, Spectral Quantum Efficiency Measurements on Cs-K-Sb Photocathodes for the Energy-Recovery Linac Test Facility bERLinPro, Master's thesis, HumboldtUniversität zu Berlin, 2017.

[26] S. Tanuma, C. J. Powell, and D. R. Penn, Calculations of electron inelastic mean free paths. IX. Data for 41 elemental solids over the $50 \mathrm{eV}$ to $30 \mathrm{keV}$ range, Surf. Interface Anal. 43, 689 (2011).

[27] J. H. Scofield, Hartree-Slater subshell photoionization cross-sections at 1254 and $1487 \mathrm{eV}$, J. Electron Spectrosc. Relat. Phenom. 8, 129 (1976).

[28] A. H. Sommer, Photoemissive Materials (John Wiley \& Sons, Inc., 1968).

[29] S. Schubert, M. Ruiz-Osés, I. Ben-Zvi, T. Kamps, X. Liang, E. Muller, K. Müller, H. Padmore, T. Rao, X. Tong, T. Vecchione, and J. Smedley, Bi-alkali antimonide photocathodes for high brightness accelerators, APL Mater. 1, 032119 (2013).

[30] S. G. Schubert, K. Attenkofer, J. Smedley, T. Kamps, M. Schmeisser, E. Muller, M. Ruiz Osés, H. Padmore, J. Wong, and J. Xie, in Proceedings of IPAC 2015 (JACoW, Richmond, VA, 2015), pp. 2566-2568. 
[31] A. R. H. F. Ettema and R. A. de Groot, Electronic structure of $\mathrm{Cs}_{2} \mathrm{KSb}$ and $K_{2} \mathrm{CsSb}$, Phys. Rev. B 66, 115102 (2002).

[32] M. A. Mamun, C. Hernandez-Garcia, M. Poelker, and A. A. Elmustafa, Correlation of $\mathrm{CsK}_{2} \mathrm{Sb}$ photocathode lifetime with antimony thickness, APL Mater. 3, 066103 (2015).

[33] L. Cultrera, S. Karkare, H. Lee, X. Liu, I. Bazarov, and B. Dunham, Cold electron beams from cryocooled, alkali antimonide photocathodes, Phys. Rev. Accel. Beams 18, 113401 (2015).

[34] A. Menegolli, M. Prata, M. C. Prata, G. L. Raselli, and C. Vignoli, Measurement of photocathode spectral response at cryogenic temperature, Nucl. Instrum. Methods Phys. Res., Sect. A 572, 446 (2007).

[35] P. Gupta, L. Cultrera, and I. Bazarov, Monte Carlo simulations of electron photoemission from cesium antimonide, J. Appl. Phys. 121, 215702 (2017).
[36] C. Cocchi, S. Mistry, M. Schmeisser, J. Kuehn, and T. Kamps, J. Phys. Cond. Matt. (to be published).

[37] R. Nathan and C. H. B. Mee, Photoelectric and related properties of the Potassium-Antimony-Caesium photocathode, Int. J. Electron. 23, 349 (1967).

[38] G. Wang, R. Pandey, N. A. Moody, and E. R. Batista, Degradation of alkali-based photocathodes from exposure to residual gases: A first-principles study, J. Phys. Chem. C 121, 8399 (2017).

[39] L. Cultrera, H. Lee, and I. Bazarov, Alkali antimonides photocathodes growth using pure metals evaporation from effusion cells, J. Vac. Sci. Technol. B Nanotechnol. Microelectron. 34, 011202 (2016).

[40] J. Kuehn, M. Buerger, A. Frahm, A. Jankowiak, T. Kamps, G. Klemz, G. Kourkafas, P. Murcek, A. Neumann, N. Ohm, M. Schmeisser, M. Schuster, J. Teichert, and J. Voelker, in Proceedings of IPAC 2018 (JACoW, Vancouver, BC, 2018), pp. 1247-1250. 